\title{
Relationship between light and dark period activity cycles and oral condition in senescence-accelerated mice
}

\author{
By \\ Mitsuo IINUMA ${ }^{1}$, Sayoko YASUI ${ }^{1}$, Mika OONISHI ${ }^{1}$, Chika KURATA $^{1}$, \\ Yukiko ICHIHASHI ${ }^{1}$, Yasuo TAMURA ${ }^{1}$ and Kin-ya KUBO ${ }^{2}$ \\ ${ }^{1}$ Department of Pediatric Dentistry, Asahi University School of Dentistry, 1851-1 Hozumi, Mizuho, Gifu, 501-0296, Japan \\ ${ }^{2}$ Seijoh University Graduate School of Health Care Studies, 2-172 Fukinodai, Tokai, Aichi, 476-8588, Japan \\ - Received for Publication, January 25, 2011 -

\begin{abstract}
Key words: light and dark period, activity cycles, tooth extraction, soft food, SAMP1
Summary: We investigated the effect of tooth absence and masticatory abnormalities due to powdered food feeding starting during the juvenile period on light and dark period activity cycles in senescence-accelerated mice (SAMP1) . SAMP1 were divided into 5 groups: Group 1, maxillo-mandibular molar tooth extraction; Group 2, maxillary molar tooth extraction; Group 3, mandibular molar tooth extraction; Group 4, powdered food; and Group 5, sham-operated control. Senescence was observed earliest in the powdered food group. Total 24-hour activity was higher in the control group than in the four other groups. In the powdered food group, the dark period activity decreased to less than $60 \%$ of the total activity in the 36th week. In the tooth extraction groups (Groups 1-3), dark period activity decreased to less than $60 \%$ of the total activity in the 40th week. The control group dark period activity remained above $60 \%$ for the entire experimental period. Thus, the distinction between the light and dark periods disappeared earlier in the four experimental groups compared with the control group. Significant correlations were noted among total activity, degree of senescence, and percent dark period activity in each experimental group. Functional masticatory insufficiency promoted dementia and behavioral abnormalities in SAMP1.
\end{abstract}

\section{Introduction}

The aging of the Japanese society is associated with an increase in the number of patients with senile dementia, and provisions to deal with this problem are becoming increasingly important. There is a clear relationship between the oral condition and general health ${ }^{1,2)}$, but the relationship between oral conditions with aging and dementia remains unclear ${ }^{3)}$. In the present study, we used senescenceaccelerated mice (SAMP1) to evaluate the effects of tooth absence and masticatory abnormalities induced during the juvenile period on life cycle and activity at later ages during which peripheral symptoms of dementia appear. Rodents, however, are nocturnal animals with higher activity levels in the dark period than in the light period, showing an activity cycle that follows the biological clock, and diurnal activity levels in senile rats aged 90 to 108 weeks is significantly shorter than that in juvenile rats, with no differences in the activity levels between the light and dark periods. Further, spontaneous activity is decreased, indicating that the nocturnal behavior of senescent animals is disturbed $^{4}$. In the present study, this characteristic was used as an index of dementia.

\section{Materials and Methods}

Fifty male SAMP1 mice were used. The strain was kindly donated by the Institute of Frontier Medical Sciences, Kyoto University. We bred brother-sister in the animal facility of the Central Research Division at the School of Dentistry, Asahi University. The SAMP8 mouse has an average life span of 12 months (about half life span of normal mice) and begins to show deficits in learning and memory and aging at 5 months. The characteristics of this strain were described in detail by Takeda et al. ${ }^{5,6}$. The animals were bred and maintained under conventional conditions; housed in groups of 5 in plastic cages $(175 \mathrm{~W} \times$ $245 \mathrm{D} \times 125 \mathrm{H} \mathrm{mm})$ under temperature $\left(23 \pm 1^{\circ} \mathrm{C}\right)$, humidity $(55 \pm 2 \%$ ), and light (12 h; light period, 6:00-18:00; dark period, 18:00-6:00) controlled conditions; and water was available ad libitum. This experiment was conducted

Department of Pediatric Dentistry, Asahi University School of Dentistry, 1851-1 Hozumi, Mizuho, Gifu, 501-0296, Japan. E-mail: iinuma@dent. asahi-u.ac.jp

This study was supported by a grant-in-Aid for Scientific Research (No. 20592420) from the Ministry of Education, Science, Sports and Culture in Japan, 2008-2011. 
according to the Animal Experiment Guidelines established by Asahi University.

The molars were extracted in 30 of the 50 mice. Five weeks after birth, mice were fixed in a supine position for tooth extraction under anesthesia using pentobarbital $(20 \mathrm{mg} / \mathrm{kg}$ intraperitoneal injection) and divided into 3 groups of 10 mice each (Group 1, maxillo-mandibular tooth extraction group; Group 2, maxillary tooth extraction group; and Group 3, mandibular extraction group). Careful extraction of the first, second, and third maxillary molars was performed using excavators. Extraction of the first, second, and third mandibular molars was performed using auditory ossicle forceps. Following extraction, the mice were fed solid food. The remaining 20 mice received only anesthesia and underwent the surgical procedure without tooth extraction 5 weeks after birth. Thereafter, 10 of these 20 mice were fed powdered food produced by powdering solid food pellets (Group 4, powdered food group), and the other 10 mice were fed solid food (Group 5 , control group). After awakening from the anesthesia, the mice were individually placed in a polycarbonate cage with a stainless wire net cover, and housed under conventional conditions.

Beginning at the age of 4 weeks, the degree of senescence was measured every 4 weeks, based on the criteria for determining the degree of senescence put forth by the Society for the study of $\mathrm{SAM}^{7}$. The grading score system was designed to represent changes in the behavior and appearance of these mice. The eleven categories include reactivity, passivity, glossiness, coarseness, hair loss, ulcer, periophthalmic lesions, cataract, corneal ulcer, corneal opacity and lordokyphosis. In general, each category has five grades corresponding to the intensity of the changes. For example, grade 0 represents no particular changes and grade 4 represents the most severe changes. Each grade in each category is clearly defined. Furthermore, to investigate mouse activity levels, we measured locomotion in an apparatus ( $27.5 \mathrm{~cm}$ long, $43.5 \mathrm{~cm}$ wide) in which spontaneous animal locomotion was detected by interruptions of near-infrared beams. In this Locomo-sensor ${ }^{\circledR}(\mathrm{LCM}-20 \mathrm{C}$ To-yo Industry Toyama Japan), three equally spaced pairs of near-infrared beams crossed the width of the apparatus, and five equally spaced pairs of near-infrared beams crossed the length of the apparatus. The number of times a mouse interrupted one of the near-infrared beams was automatically counted as a measure of locomotion. The mice were placed in the cage at 13 o'clock, and their activity was measured every hour for 24 hours. Because activity was high immediately after being placed into the cage, the data for the first hour between 13 and 14 o'clock was excluded from the analysis. The light period was set for a total of 11 hours between 14 and 18 o'clock, and between 6 and 13 o'clock the following morning, and the amount of activity in the light period was calculated by converting it to the amount of activity over 12 hours.
Statistical evaluation was performed using analysis of variance and Pearson's correlation coefficient test.

\section{Results}

Figure 1 shows the degree of senescence every fourth week in each experimental group. Figure 2 shows the changes in the total activity for 24 hours over the experimental period. Senescence first appeared at week 16 in the powdered food group, and then in the three tooth extraction groups. The degree of senescence did not significantly differ among groups. The progression of senescence was slowest in the control group. The total amount of activity over 24 hours increased with age until the 12th week in all the groups, except in the maxillo-mandibular tooth extraction group, which exhibited decreased activity immediately after tooth extraction. The activity levels were maintained between the 12th and 28th weeks, and decreased after the 28th week. Activity was higher in the control group than in the other four experimental groups throughout the entire experimental period.

Figure 3 shows the activity over 24 hours at each age for each group. At each age, the activity was highest immediately after the mice were placed in the cage, and then gradually decreased thereafter during the initial light period, increased in the dark period, and decreased again during the second light period. In each experimental group, mice under 12 weeks of age exhibited high activity levels in the dark period, and in the first light period, whereas activity was low in the second light period. Thereafter, between 16 and 36 weeks of age, activity was low in both light periods, and high in the dark period. At the 40th week, activity in the dark period decreased in all groups except for the control group, and no differences in activity were noted thereafter between the light and dark periods. In the control group, however, the light and dark period activity cycles were observed until the 56th week, at which point differences in activity between the light and dark periods gradually disappeared. Figure 4 shows the changes in the ratio of activity in the dark period to total 24-hour activity in each experiment group. In each group, starting in the juvenile stage, activity during the dark period accounted for $60 \%$ to $70 \%$ of the whole activity. In the control group, the dark period activity decreased to less than $60 \%$ that of the total activity at the 56th week, and differences in activity levels between the light and dark periods disappeared in the 60th week. In the powdered food group, dark period activity decreased to less than $60 \%$ of the total activity at the 36th week, and the activity in the dark period became almost the same as that in the light period after the 50th week. In the tooth extraction groups, dark period activity decreased to less than $60 \%$ at the 40 th week, and differences in the light and dark periods disappeared at 48 to 52 weeks, earlier than that of the control group. 


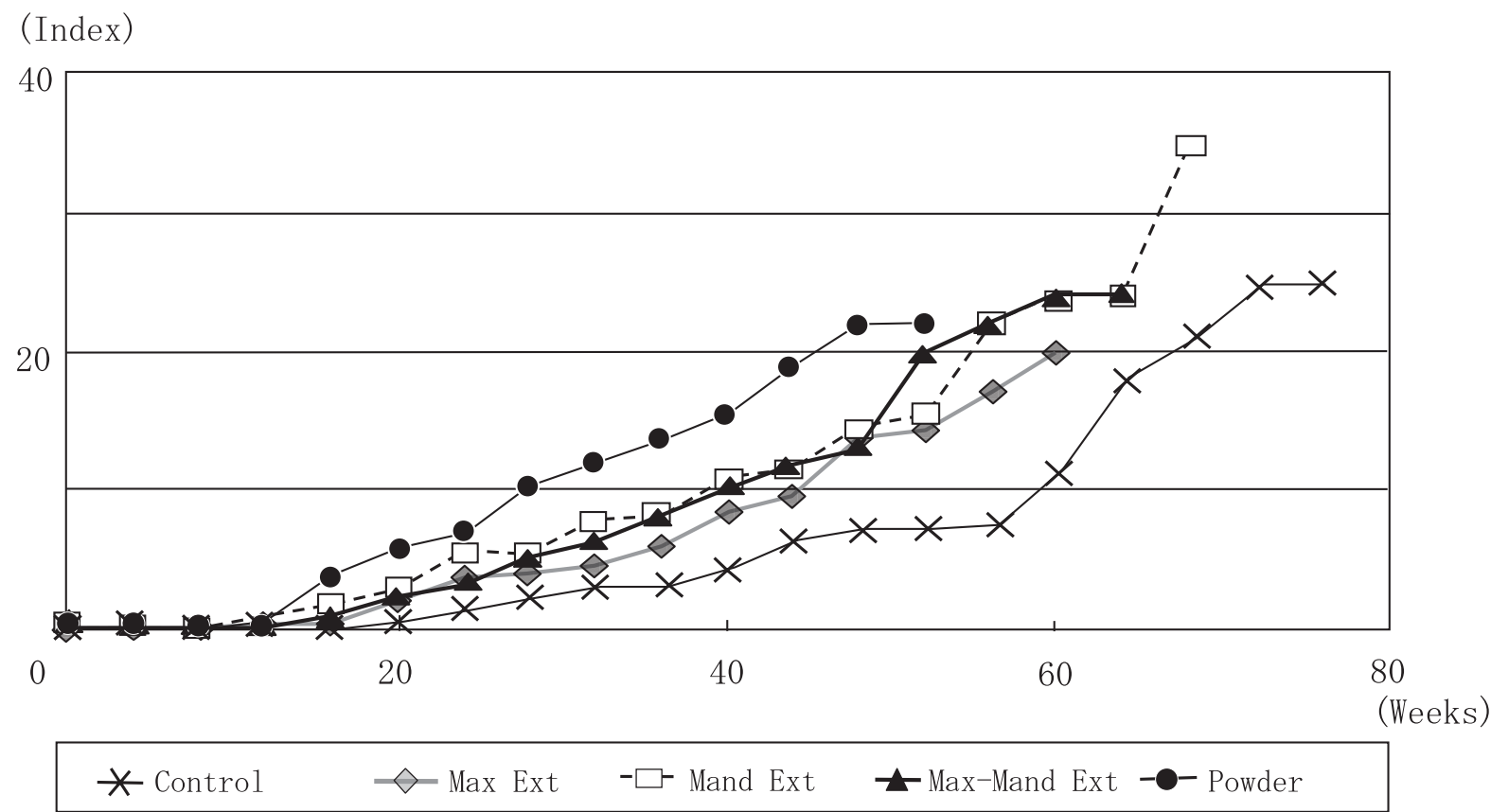

Fig. 1. Changes in the degree of senescence

(activity)

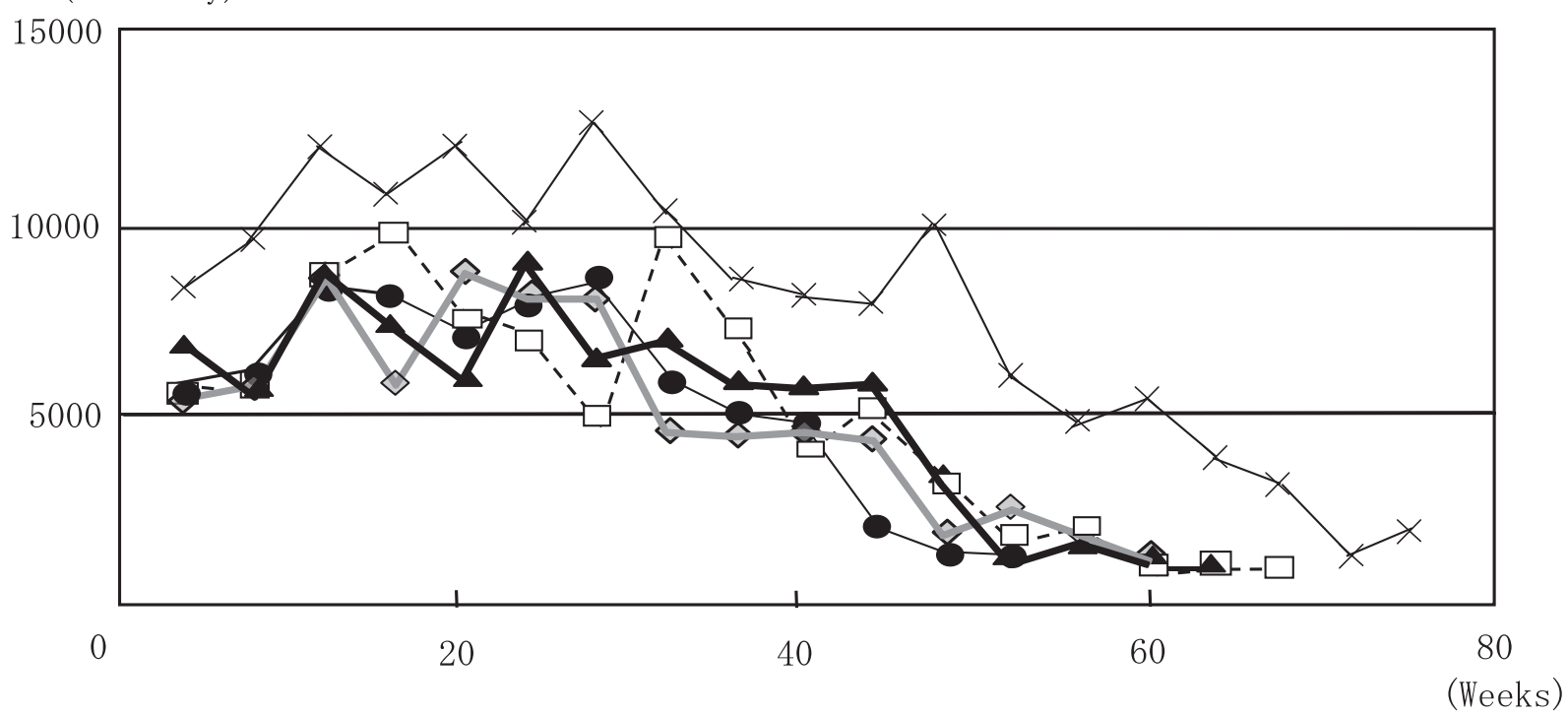

$*$ Control $\quad \nabla$ Max Ext $\quad \square$-Mand Ext $\mathbf{t}$-Max-Mand Ext $-\mathbf{0}-$ Powder

Fig. 2. Changes in the total activity for 24 hours 

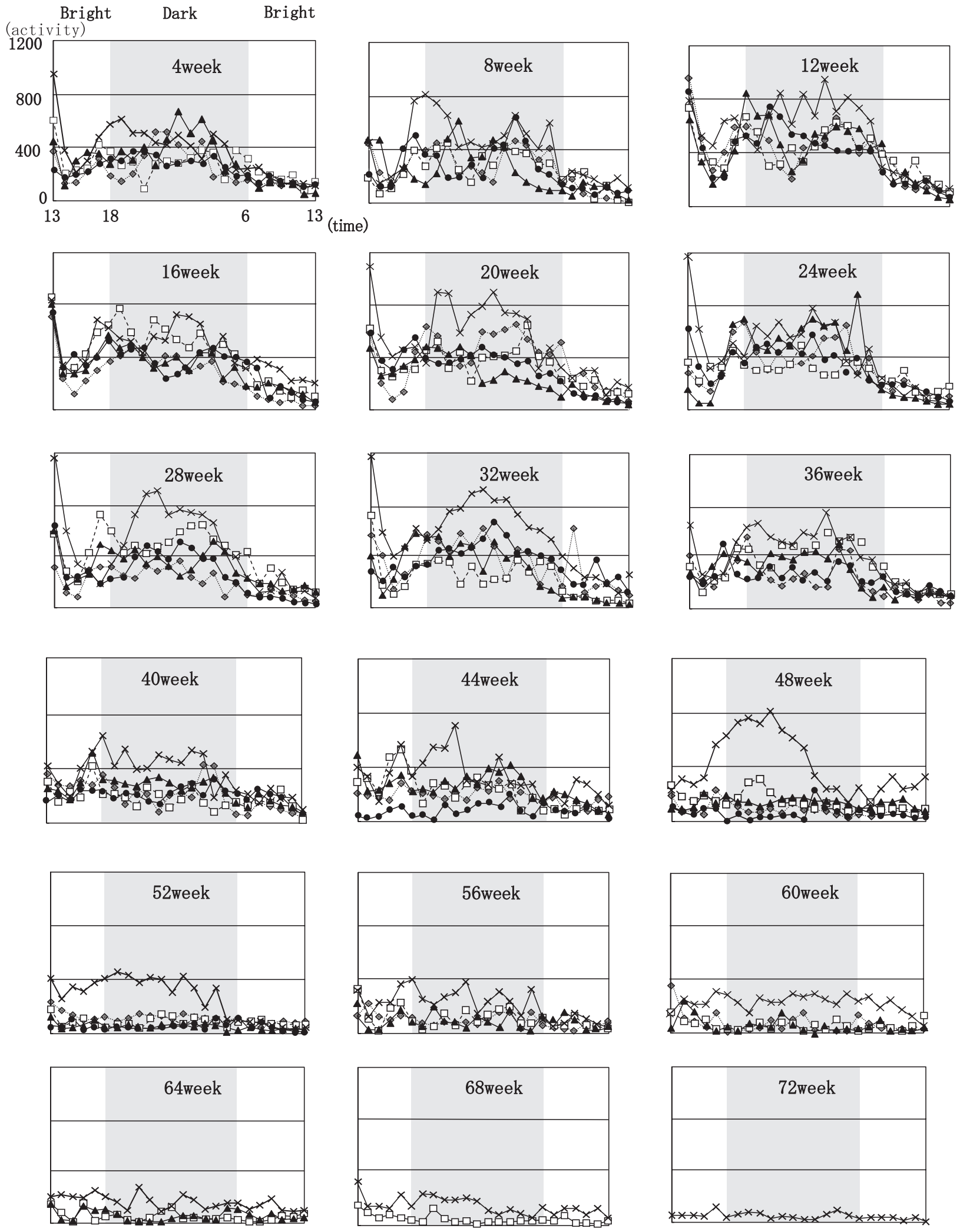

* Control

$\therefore \quad$ Max Ext $\square \quad$ Mand Ext $\_$Max-Mand Ext -2 Powder 


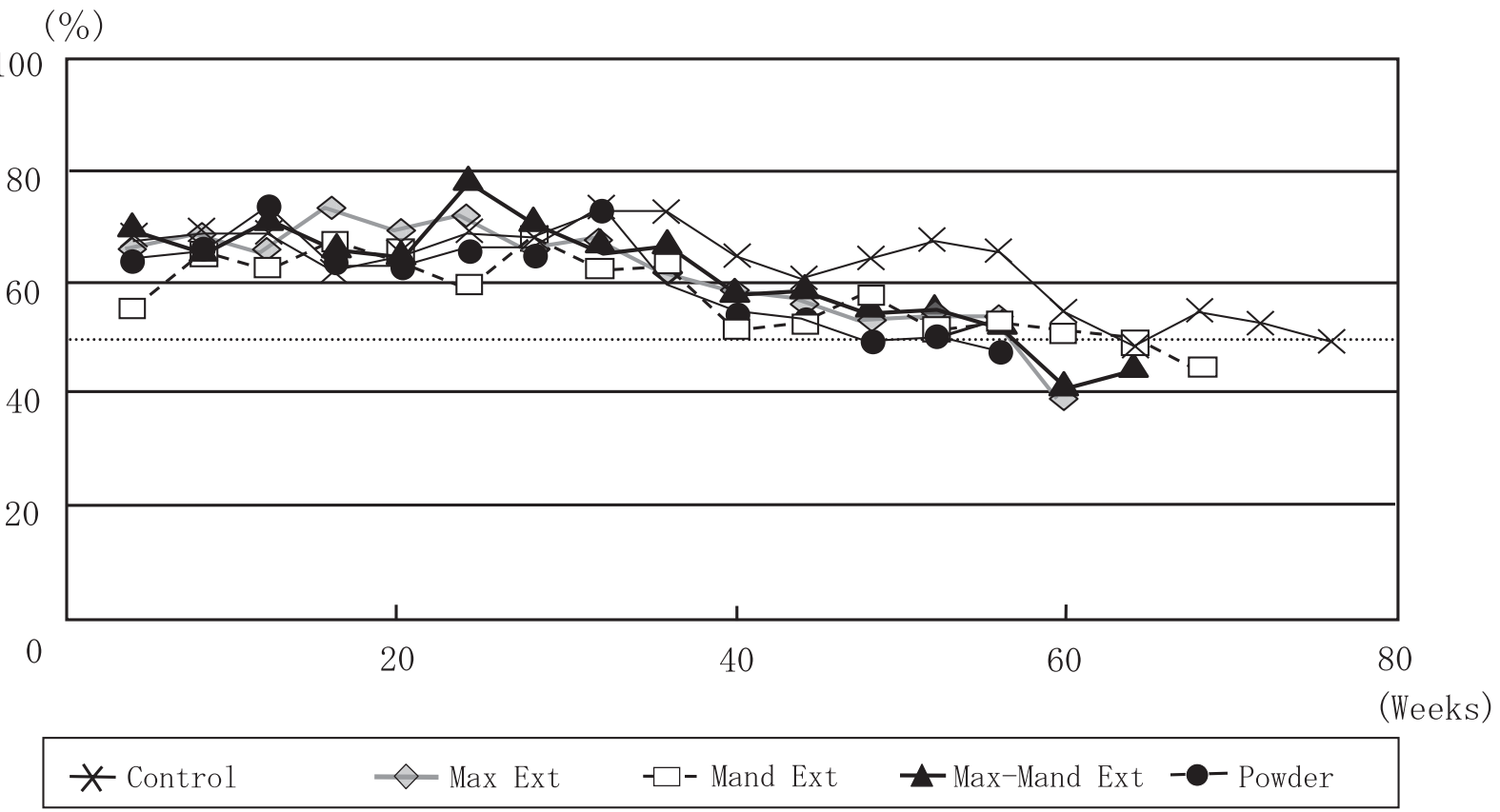

Fig. 4. Changes in the ratio of the activity in the dark period to that during the whole day in each group

\section{(activity)}

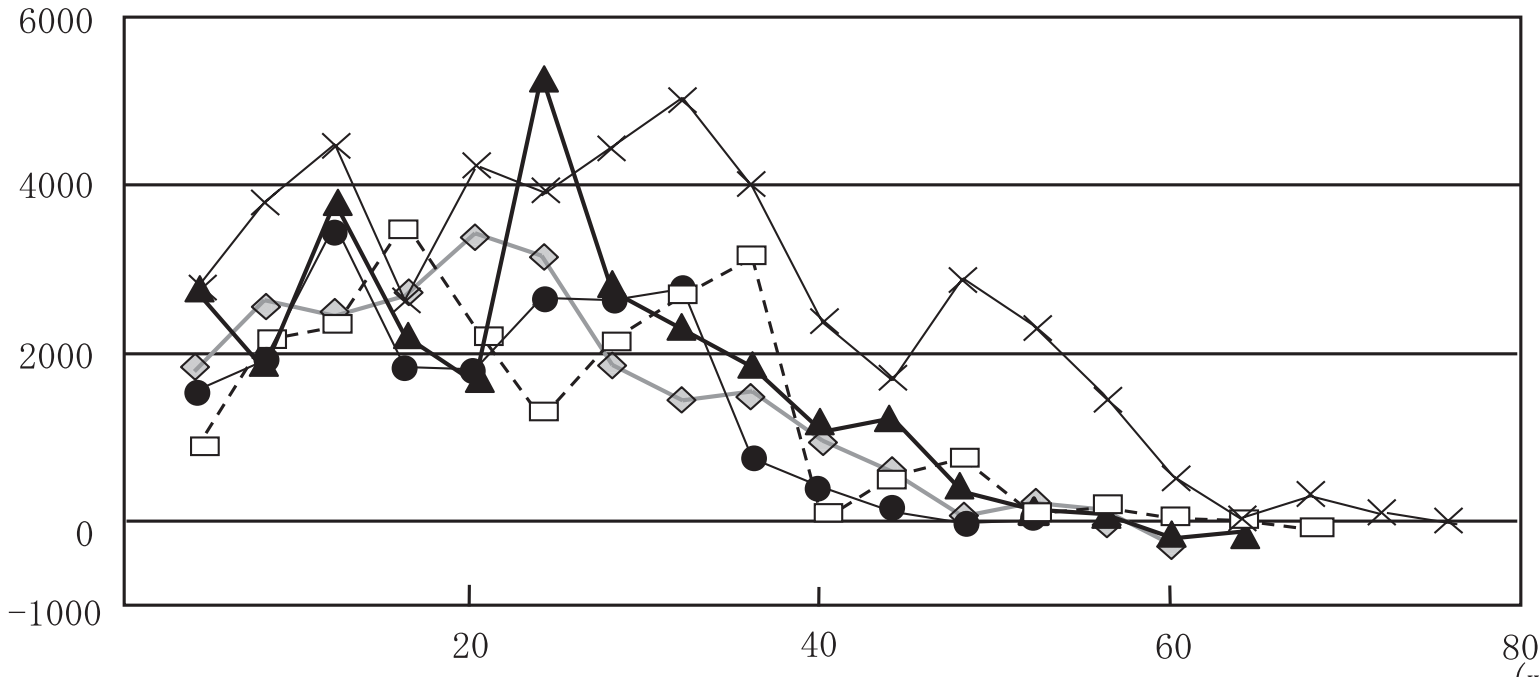

(weeks)

$*$ Control $\quad \nabla$ Max Ext $\quad \square$ - Mand Ext $\rightarrow-$ Max-Mand Ext $-\mathbf{-}$ - Powder

Fig. 5. Differences in activity between the light and dark periods

Figure 5 shows the change in the differences in activity between the light and dark periods. The control group had the greatest difference in activity between the light and dark periods at almost every age.
Figure 6 shows the changes in the activity during the light and dark periods. The total number of beam breaks in the light period ranged from 2000 to 4000 until the 40th week, at which point the count decreased to approximately 
(A)

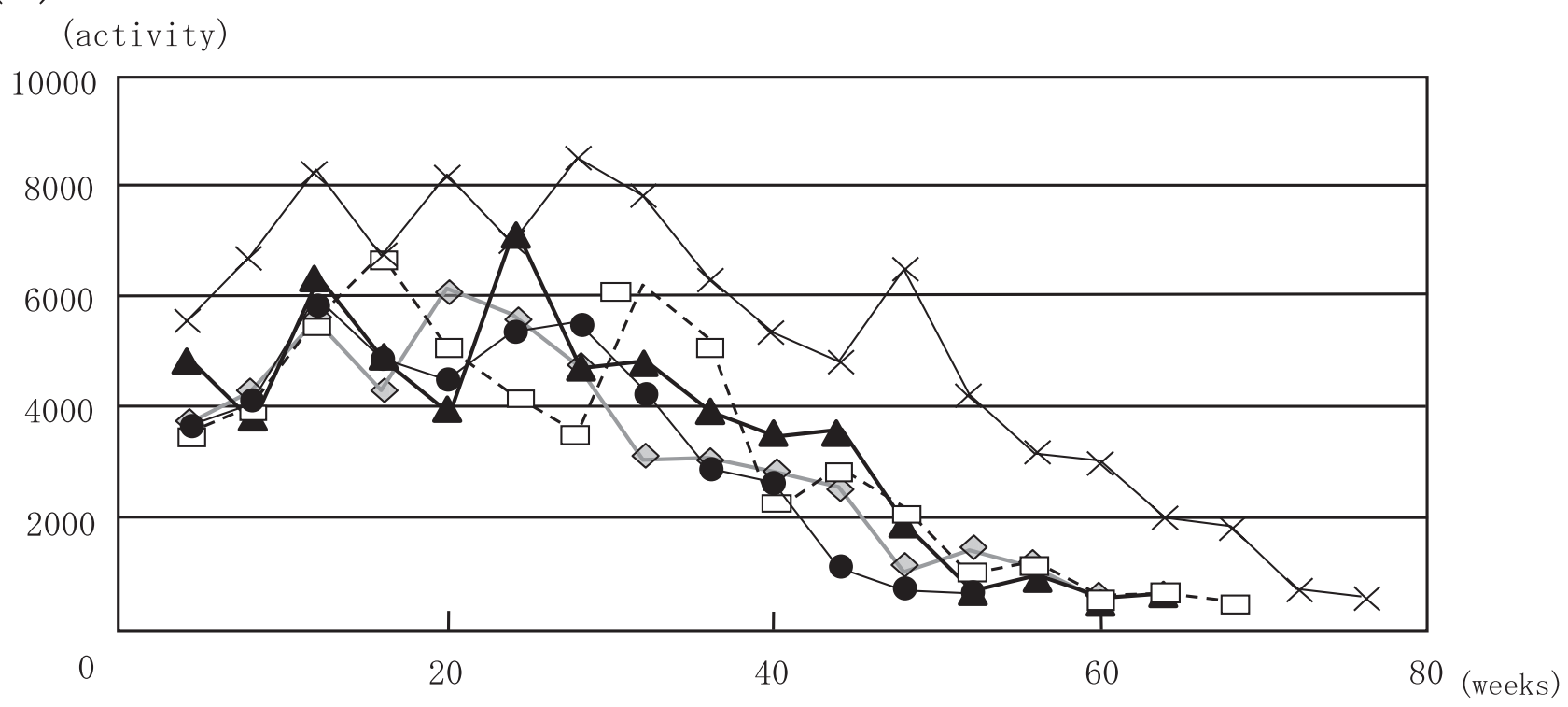

(B) (activity)

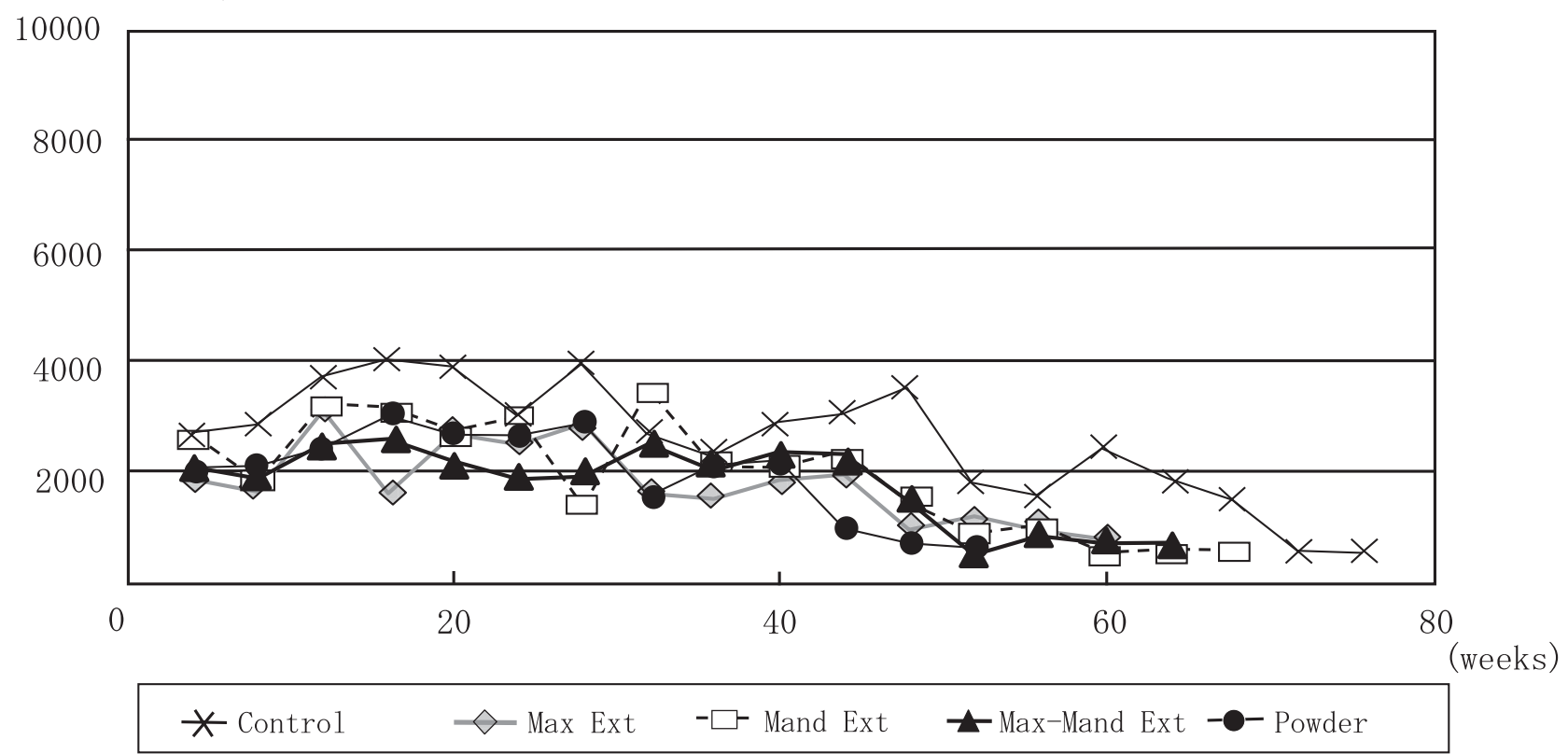

Fig. 6. Changes in the activity during the light (A) and dark periods (B) 
Table 1. Relationship between total activity, degree of senescence and percent dark period activity

\begin{tabular}{|c|c|c|c|}
\hline & $\begin{array}{c}\text { Total Activity } \\
\text { Ratio of dark period activity }\end{array}$ & $\begin{array}{c}\text { Ratio of dark period activity } \\
\text { Degree of Senescence }\end{array}$ & $\begin{array}{c}\text { Total Activity } \\
\mid \\
\text { Degree of Senescence }\end{array}$ \\
\hline Control & $0.38^{* *}$ & $-0.53^{* *}$ & $-0.62^{* *}$ \\
\hline Max Ext & $0.26^{\star}$ & $-0.62^{* *}$ & $-0.47^{\times *}$ \\
\hline Mand Ext & $0.24^{*}$ & $-0.44^{* * x}$ & $-0.41^{* *}$ \\
\hline Powder & 0.16 & $-0.26^{\star}$ & $-0.45^{\text {*x }}$ \\
\hline
\end{tabular}

$※: \mathrm{p}<0.05 * *: \mathrm{p}<0.001$

1000. The number of beam breaks in the dark period was greater than 4000 until the 30th week in every group, and then decreased to less than 2000 at the 50th week, and further decreased to approximately 1000 thereafter in all four experimental groups.

Table 1 shows the relationships among total activity, degree of senescence, and percent dark period activity (activity rhythm). Significant correlations were observed among the three items in every group.

\section{Discussion}

The present study was performed to examine the effect of long-term masticatory insufficiency on senescence. Tooth extraction was performed at 5 weeks after birth, immediately after weaning, and thus the mice lacked masticatory experience.

Activity levels were high for the first hour after being placed in the apparatus at every age. We considered that this initial high level of activity was a result of the novelty of the environmental change; therefore, the data for the first hour between 13 and 14 o'clock were excluded from the analysis.

Although activity was high in the light period until 18 o'clock before 12 weeks of age, we considered that this was because an activity rhythm had not been sufficiently established in the juvenile period, and the mice needed time to adapt to the environment. The difference in the activity level after 12 weeks of age between the first light period and the second light period was considered to be due to the effect of the change in the environment.

There are several patterns of animal behavior, such as biorhythm, and animals have the ability to adapt to external changes that repeat with regularity. This is under control of a biological clock, and the circadian rhythm is the most universal rhythm in animals. Browman ${ }^{8}$ bred rats for 25 generations under constant light conditions, and reported that their activity continued to fluctuate with a 24hour cycle. Aschoff ${ }^{9)}$ reported that rats bred for three generations under constant light or constant dark condi- tions maintained the circadian rhythm of their activity. Richter ${ }^{10)}$ investigated activity levels in congenitally blind rats and in rats that were blinded immediately after birth, and reported that their 24-hour cycle activity continued after birth. These findings suggest that even in mice born and bred under constant light or constant dark conditions, the circadian rhythm of their activity remained, and the effects of the biological clock are inherent.

In the control group in the present study, the light and dark period activity cycle was apparent already in the juvenile stage, whereas the distinction between the light and dark period activity cycle decreased in the senile stage, showing disorders of the biological clock. Although the activity in the control group decreased in both the light and dark periods, because the decreases in the activity were more marked in the dark period than in the light period, the ratio of activity in the light period also decreased. Visual disorders due to aging are not likely the cause of this change. Nabeshima et al. ${ }^{4}$ reported no differences in the selection rate of dark places between juvenile and senile groups and Kato et al. ${ }^{11)}$ reported that 8 to 13-month old pre-senile hamsters could adjust to various light and dark period cycles. Rosenberg ${ }^{12)}$ reported that senile hamsters showed phase displacement of activity induced by optical pulses, similar to juvenile hamsters, with few aging-related changes in sensitivity to light. Furthermore, Yamaoka et al. ${ }^{13)}$ reported that the effect of inversion of the light and dark period cycle on behavioral rhythm is also observed in rats with cataracts; therefore, disorders in the activity cycle due to aging are not caused by disorders in optical input pathways (visual disorders), but are caused by changes in the clock mechanism itself.

The biological clock is located in the suprachiasmatic nucleus in the hypothalamus, and the expression of c-fos gene, induction of fos protein, and some types of peptides are decreased in the suprachiasmatic nucleus, in aged animals ${ }^{14,15)}$. Shibata et al. ${ }^{14)}$ reported that vasoactive intestinal peptide release in the suprachiasmatic nucleus, which has an important role in adapting to external circumstances, is attenuated by aging. Based on these findings, we considered that our results are due to a decrease in peripheral 
information processing with aging, and thus information transmission input in the tooth extraction area is decreased due to tooth loss, causing disorders in the light and dark period cycle. A functional network is established in the central nervous system, and synapses are the most important information transmission sites in the network; therefore, if information is not transmitted via synapses, the information is not transmitted to the next cell. Furthermore, cells that do not receive information for a long period undergo apoptosis ${ }^{16)}$. Onozuka et al. ${ }^{17)}$ reported that the number of pyramidal cells in the hippocampus CAI area in senile mice that had undergone maxillary molar grinding, extraction, or amputation of the masseteric nerve, decreased to $77 \%$ of the level in mice at the same age with normal molars, suggesting that decreases in the number of pyramidal cells was caused by functional masticatory insufficiency and resulted in cell death. Therefore, we consider that decreases in masticatory function in the senile period further facilitate nerve cell death in the hippocampus with aging, and this phenomenon might be due to changes in the suprachiasmatic nucleus.

Activity in the light and dark period cycle was not significantly different between the control and experimental groups until the 40th week, when senescence began to be exhibited to some extent. Based on the results of watermaze experiments to determine the relationship between tooth grinding and memory, Onozuka et al ${ }^{18)}$ reported that juvenile mice showed no differences in the time required to reach the escape platform between tooth grinding and non-grinding groups, whereas senile mice in the toothgrinding group showed an increase in the escape latency, indicating a decreased ability to learn the task, which is consistent with the results of the present study. These results suggest that although functional masticatory insufficiency at an early age gradually progresses to senescence, it has little influence on behavior and memory in the early period, and a rapid influence is observed after a certain period.

The behavioral effects of tooth loss and eating powdered food are considered to be due to stress. Squire et $a l .{ }^{19)}$ reported that stress induces neuronal death in the hippocampus, and Onozuka et al. ${ }^{17)}$ reported that increases in the corticosterone concentration are induced by maxillary molar grinding. Therefore, the functional masticatory insufficiency in these mice may be due to chronic stress.

\section{Conclusion}

Our experiments revealed that functional masticatory insufficiency promoted the occurrence of dementia and behavioral abnormalities in SAMP1.

\section{References}

1) Saito $T$ and Hasegawa K: Change of type of dietand ADL in aged patients after dental treatment. J Jap Gerodont 2003; 17:15-20. (in Japanese)

2) Saito $\mathrm{T}$ and Hasegawa K: Clinical investigation of five aged patients who showed the improvement of ADL after dental treatment. J Jap Gerodont 2003; 17:321-325. (in Japanese)

3) Okimoto K, Ieiri K, Matsuo K and Terada Y: Aging and mastication; The relationship between oral status and the progress of dementia at senile hospital. J Jap Prothodont Soc 1991; 35:931-943. (in Japanese).

4) Nabeshima T, Hasegawa M, Nakayama S, Kinoshita H, Amano M and Hasegawa T: A study of emotional behavior of senile dementia by aging rats: Jpn. J. Psychopharmacol 1993; 13:65-72. (in Japanese)

5) Takeda T, Hosokawa M, Takeshita S, Irino M, Higuchi K, Matsushita T, Tomita Y, Yasuhira K, Hamamoto H, Shimizu K, Ishii M and Yamamuro T: A new murine model of accelerated senescence. Mech Aging Dev 1981; 17:183-194.

6) Takeda T, Hosokawa M, Higuchi K, Hosono M, Akiguchi I and Katoh H: A novel murine model of aging, Senescence-Accelerated Mouse (SAM). Arch Gerontol Geriatr 1994; 19(2):185-192.

7) Hosokawa M, Sakai R, Higuchi K, Takeshita S, Shimizu K, Hashimoto H, Honma A: A method of evaluation of the degree of senescence in senescence accelerated mouse (SAM). Mech Ageing Dev 1984; 26:91-102.

8) Browman LG: Artifical Sixteen-hour day activity rhythms in the white rat. Amer J Physiol 1952; 168:694-697.

9) Aschoff J: Tagesperiodik bei mäusestämmen unter konstanten umgebungs-bedingungen. Pflügers Arch Bd 1955; 262:S.51-59.

10) Richter CP: Inborn nature of the rat's 24-hour clock. J Comp Physiol Psychol 1971; 75:1-4.

11) Kato $T$, Usami $T$, Noda $Y$, Hasegawa $M$, Ueda $M$ and Nabeshima $T$ : The effects of the loss of molar teeth on spatial memory and acetylcoline release from the parittal cortex in aged rats. Behavi Brain Res 1997; 83:239-242.

12) Rosenberg RS, Winter JB, and Rechtschaffen A: Effect of light cycle phase reversal on sleep rhythms of young and old rats (Abstr) Sleep Res 1980; 9:105.

13) Yamaoka S: Changes of circadian rhythm by aging: Clinic Psychatry 1955; 24:641-651. (in Japanese)

14) Shibata $S$ and Ooshima I: Neurotransmitter and aging: Clinic. Psychatry 1955; 24:633-639. (in Japanese)

15) Sutin EL, Dement WC, Heller HC and Kilduff T: Light-induced gene expression in the suprachiasmatic nucleus of young and aging rats. Neurobiol Aging 1993; 14:441-446.

16) Vanický I, Maršala M, Orendácová J and Maršala J: Silver Impregnability of ischemia-senssitive neocortical neurons after $15 \mathrm{~min}$ utes of cardiac arrest in the dog. Anat. Embryol (Berl) 1992; 86:167-173.

17) Onozuka M: Impairment of spatial memory and changes in astrogrial responsiveness following loss of molar teeth in aged SAMP8 mice. Behav. Brain Res 2000; 108:145-153.

18) Onozuka M, Watanabe K, Mirbod SM, Ozono S, Noshiyama K, Karasawa N and Nagatsu I: Reduced mastication stimulates impairment of spatial memory and degeneration of hippocampal neurons in aged SAMP8 mice. Brain Res 1999; 826:148-153.

19) Squire LR: Memory and the hippocampus - A synthesis from findings with rats, monkeys, and humans -. Psychol Rev 1992; 99:195-231. 